areas like prevention, as well as new materials and techniques, it may become more difficult to train dental students in clinical aspects like crown and bridgework or endodontics with the same standards as in the past. ${ }^{4}$

Acknowledgements: We would like to thank Dr German F. Alvarado for his comments.

N. A. Araujo, S. P. Behrens, Lima, Peru

1. Oxley C J, Dennick R, Batchelor P. The standard of newly qualified dental graduates - foundation trainer perceptions. Br Dent J 2017: 222: 391-395.

2. Farzianpour F, Monzavi A, Yassini E. Evaluating the quality of education at Dentistry School of Tehran University of Medical Sciences. Dent Res J (Isfahan) 2011; 8: 71-79.

3. Garcia R I, Sohn W. the paradigm shift to prevention and its relationship to dental education. J Dent Educ 2012; 76: $36-45$.

4. Cabot $L B$, Radford D R. Are graduates as good as they used to be? Br Dent J 1999; 186: 318-319.

DOI: $10.1038 /$ sj.bdj.2017.693

\section{Oral health}

\section{Dental neglect on wards}

Sir, during my years of clinical exposure in London as a medical student I have rotated through a range of medical specialties. I have noted that as medical professionals our duty to provide holistic care to our patients is one that is often well reflected in our ward assessments of new inpatients. We are actively encouraged to ask a complete social history, to assess mobility, and to assess mood.

However, it has come to my attention that there is a fundamental neglect of dental care across wards. Speaking with fellow colleagues the only identifiable assessment of regularly documented oral care is the pre-operative assessment checklist for theatre and radiology, which checks for dentures or crowns. Could it be that we have demedicalised oral healthcare? We regularly comment on dentition in our simple cardiology, respiratory, and abdominal examinations but for some reason this is not carried through into the patient's notes. Should we not be documenting important negative oral findings? It is not unreasonable to suggest that we are avoiding a simple opportunistic form of health promotion.

The limited literature available does suggest that poor oral health is common in older people admitted to hospital acute care wards. ${ }^{1}$ A recent evidence summary highlights the link between oral health and pulmonary disease, in particular pneumonia, demonstrating how its incidence can be lowered by implementing simple oral hygiene measures, such as offering patients chlorhexidine mouth wash and ensuring they brush their teeth. ${ }^{2}$ If applied in the hospital setting this could impact on the high number of patients developing pneumonia. We already assess venous thromboembolic risk, risk of falls and a combination of lifestyle factors. Perhaps it is time we stop turning a blind eye to oral health. Simple measures such as a dental hygiene check box on admission forms and drug/observation charts could potentially be an effective low-cost intervention with the potential to improve our patient care.

R. S. Randhawa, London J. S. Chandan, T. Thomas, Birmingham

1. Gibney J M, Wright C, Sharma A, D'Souza M, Naganathan $\mathrm{V}$. The oral health status of older patients in acute care on admission and Day 7 in two Australian hospitals. Age Ageing 2017; 25: 1-5.

2. Manger $D$, Walshaw M, Fitzgerald $R$ et al. Evidence summary: the relationship between oral health and pulmonary disease. Br Dent J 2017; 222: 527-533.

DOI: 10.1038/sj.bdj.2017.694 